\title{
Geographic Pattern as a Determinant Factor of Interlinking Climatic and Hydrological Components of the Natural Resources
}

\author{
Ekaterina V. Gaidukova *, Victor V. Kovalenko \\ Department of Hydrophysics and Hydrological Forecasts, Russian State Hydrometeorological \\ University (RSHU), Saint-Petersburg, Russia \\ ”oderiut@mail.ru
}

Keywords: fractal dimension of runoff series, coefficient of kurtosis, norm of air temperature.

\begin{abstract}
Some of the practical implications of the previously identified geographic patterns linking climate rate of surface temperature with the fractal dimension of a multi-year series of runoff, are presented. The statistical data, showing a role of coefficient of kurtosis at classification of distributions of density of probability in a range of air temperatures from -5 to $27^{\circ} \mathrm{C}$ are given. It is empirically established that statistical distributions of long-term average annual runoff series for the temperature zone with prevailing high values of the fractal dimension correspond to betadistributions of type II and when plotted on the K. Pearson diagram this type is distinguished from probability density curves in other temperature zones by kurtosis (statistically significant). It is revealed that ignoring the coefficient of kurtosis means not taking into account the climatic component of natural resources. The results of the study can be used to assess the reliability of hydraulic structures and in the field of water management.
\end{abstract}

\section{Introduction}

According to the annual water balance equation for a river basin, $X=Q+E \pm \Delta U$ (here, $X$ is precipitation; $Q$ - runoff; $E$ - evaporation; $\Delta U$ - change in the soil moisture content), the basin response to external impact (resource $X$ ) can be described statistically by a three-dimensional probability density $p(Q, E, \Delta U)$. Due to a number of reasons (the practical need for only statistically probable discharges and the lack of network observations on evaporation and soil moisture content), only one-dimensional $p(Q)$ projections are used, which is officially established in regulatory documents [1]. This entails problems related to the instability of moments of one-dimensional probability distributions, due to the presence of multiplicative noise in the stochastic model of longterm runoff formation (Fokker-Planck-Kolmogorov - FPK equation), which simulate the influence of discarded (explicitly) phase variables $E$ and $\Delta U$. This is displayed visually as the polymodality appearing in $p(Q)$ distributions or the presence of heavy-tailed distributions [2,3].

To mitigate this instability, a methodology [2] was developed that relied on the unloading of the multiplicative noise component by increasing dimensionality of the phase space where the flow formation is modelled. To perform fractal diagnosis, we used the correlation dimension recovered from the measurements of only one phase variable, according to the Takens theorem [4, 5], applied to 756 annual runoff series (predominantly, rivers of the Northern hemisphere) and detected (see [6]) a previously unknown regular pattern, the parabolic dependence of the fractal dimension on the climatic norm of the surface air temperature (Fig. 1,a). It was found that within the temperature range of $1.6-7{ }^{\circ} \mathrm{C}$, all three phase variables contribute (statistically significant) to the runoff formation in approximately one-third of river basins (Fig. 1,b). 


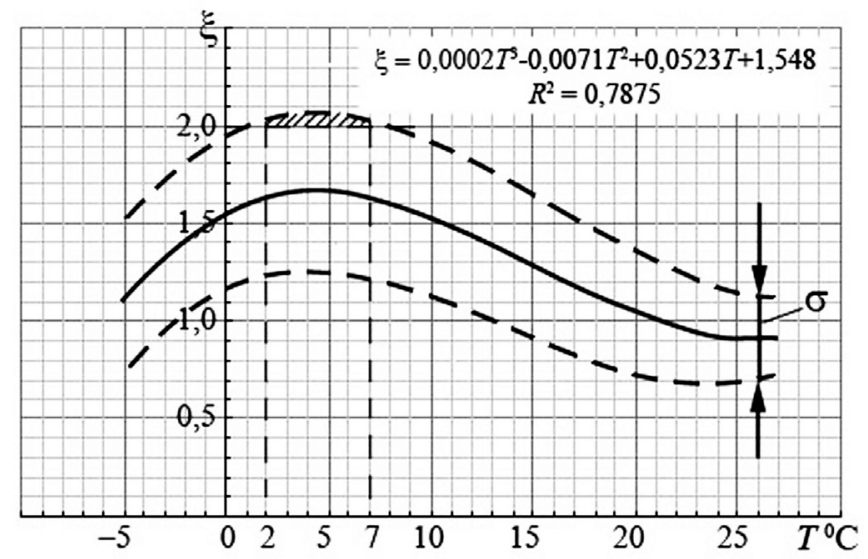

a

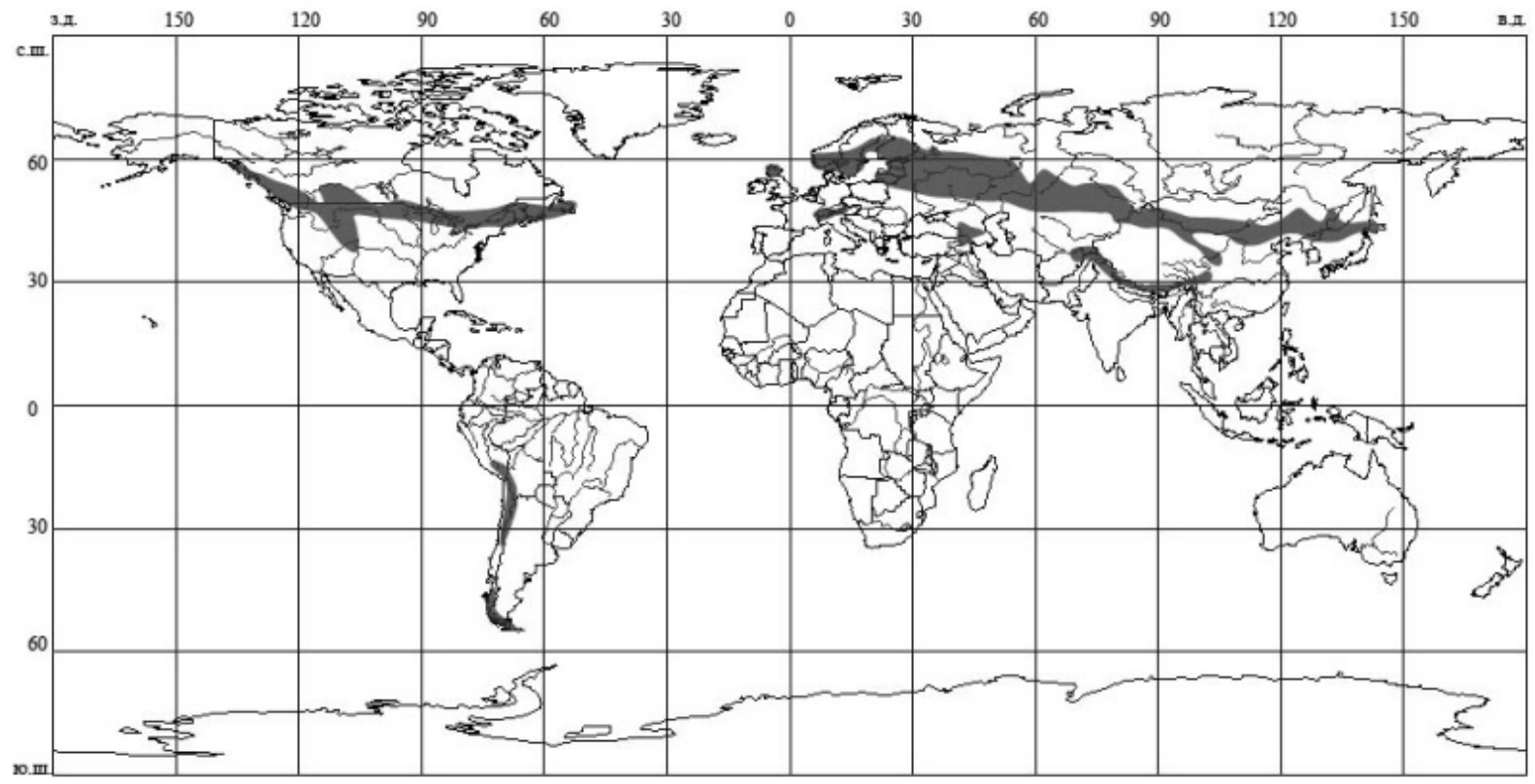

$\mathrm{b}$

Figure 1. Dependence of the fractal dimension of the long-term average annual runoff series on the climatic norm of air temperature (a) and the most probable regions with unsteady moments of onedimensional discharge distributions (grey colour) (b).

This does not imply that fractal dimensions greater than two cannot occur in another temperature range, but they are more frequent in this range and make, on average $31 \%$. Also, this does not mean that soils beyond this temperature range do not participate in the runoff formation their influence just cannot be traced under the current accuracy of fractal diagnosing.

Discovery of this pattern raises at least two questions: 1) Where is that flow formation model, which leads to a three-dimensional distribution of $p(Q, E, \Delta U)$ ? 2) Any pattern reflects the essentials of some phenomenon, which should somehow show itself due to its phenomenology nature. How does the phenomenon, whose essentials are reflected in the identified pattern, manifest itself?

To answer the first question, we need to generate a model of long-term runoff formation, which: 1) would provide the evolution of the $p(Q, E, \Delta U)$ distribution as its solution; 2) when the independent role of evaporation and soil moisture content in the runoff formation is ignored, it would be reduced to a model for one-dimensional distribution of $p(Q)$, which in case of stationary random processes (current "regulatory hydrology" represents the river runoff in such a manner) would turn into the Pearson equation, which gives a family of solutions including the Pearson 
curves of type III recommended by the Set of rules 33-101-2003 (Set of Rules, 2004), and their modification with a fixed left boundary of $p(Q=0)=0$, proposed by S. N. Kritsky and M. F. Menkel [7]. Actually, a 3-dimensional FPK equation should be produced. This problem was solved in the study [8].

The objectives of this article are as follows: a) to identify the "one-dimensional" phenomenological consequences of the model solution for $p(Q, E, \Delta U)$ and find confirmation that within the range $1.6-7{ }^{\circ} \mathrm{C}$ they are not less than $30 \%$ and that this figure is greater than in other temperature ranges (below $1.6{ }^{\circ} \mathrm{C}$ and above $7{ }^{\circ} \mathrm{C}$ ); b) to identify the most likely types of probability density distributions for water discharge in different temperature zones; c) to identify the hydrological component to be considered for providing interlinking between the climatic and hydrological components of natural resources.

\section{Theoretical and Empirical Foundations}

A multidimensional FPK model describing the evolution of three-dimensional distribution of probability density $p(Q, E, \Delta U)$ was obtained in the study [8]. First, it allows to simulate and predict the processes in river basins, where the fractal dimension of the runoff series is greater than two (predominantly, this is the temperature range $1.6-7^{\circ} \mathrm{C}$ ), and second, it eliminates the problems mentioned in the introduction. However, its practical implementation takes some time (parameterization technique, reduction to a statistically stationary case, selection of the optimal numerical solution methods, etc.).

The absence of such model does not prevent hydrologists from constructing one-dimensional empirical distributions of $p(Q)$ facing none of the above problems. These are just non-existent under the scope of engineering hydrology, since the latter relies only on the side of science, which asserts the surrounding reality. This assertion is performed via the actual series of runoff observations; their probability distributions are approximated by solutions of the Pearson equation and the Pearson coefficients are used as adjustable parameters to the empirical field of points on the runoff probability curves. When the instability of moments is mentioned, it means the instability of their statistical estimates due to short-term series (rather than the instability of solutions of runoff formation models in the form of a system of differential equations for the moments). The second modes of empirical distributions are considered statistically insignificant (for the same reasons, scarcity of observations).

However, all these problems become obvious as soon as we move to use the second, prognostic aspect of science. In this case, working with series is insufficient and we need a model of runoff formation. Meanwhile, fractal diagnostics indicates that probability distributions in certain geographic zones should be three-dimensional, model solutions can be two-modal and onedimensional model projections result in unstable solutions for the moments, etc.

Therefore, if the models of runoff formation are really multidimensional, the empirical material should produce the above features of these solutions, to a certain extent and in some form. This is confirmed by the table (756 series of annual average water discharges of water basins located in the northern and southern hemispheres were processed [6]). As is known, the flatness and peakedness of distributions is determined by the kurtosis $\varepsilon$ [9]. The table 1 shows that all three temperature zones have series with both positive and negative values of $\varepsilon$. However, due to the low accuracy of $\varepsilon$ calculation (for short series), it is reasonable to focus on its average (for all series and for each zone) values, which are close to zero in the northern zone and for the range of our interest are $1.6-7{ }^{\circ} \mathrm{C}-0.25$ (the material on the accuracy of the coefficient of kurtosis can be found in the source [10]). This indicates that peaked distributions dominate objectively in this range, although there are many distributions with small negative values of $\varepsilon$, i.e., with flattened or bimodal vertex. In practice, this leads to the presence of heavy-tailed distributions in this zone and the ensuing negative effects for the safety of hydraulic structures and water management in general. 
Table 1. Statistical characteristics of the runoff series.

\begin{tabular}{|l|c|c|c|}
\hline \multirow{2}{*}{\multicolumn{1}{|c|}{ Characteristics of the series }} & \multicolumn{3}{c|}{$\boldsymbol{T}\left[{ }^{\circ} \mathbf{C}\right]$} \\
\cline { 2 - 4 } & $\mathbf{- 5 ~ \ldots . ~ 1 . 6}$ & $\mathbf{1 . 6} \ldots \mathbf{7}$ & $\mathbf{7 ~ \ldots . ~ 2 7}$ \\
\hline Number of series examined & 125 & 125 & 242 \\
\hline Number of cases with positive kurtosis (in \%) & 38 & 47 & 43 \\
\hline Average kurtosis value & 0.01 & 0.25 & 0.23 \\
\hline $\begin{array}{l}\text { Number of cases (in \%) where unimodal histograms } \\
\text { were visually observed (number of columns - 7) }\end{array}$ & 63 & 75 & 52 \\
\hline Parameter $\beta_{1}$ classifying the Pearson distribution & 0.34 & 0.48 & 0.76 \\
\hline Parameter $\beta_{2}$, classifying the Pearson distribution & 3.01 & 3.25 & 3.23 \\
\hline
\end{tabular}

This is determined by the fact that under such values of distribution steepness, the number of deviations from the mean value $(\bar{Q})$ by the triple standard deviation $(\sigma)$ is significantly greater than under its zero values (for example, $\bar{Q}=785.6 ; \sigma=181.2 ; \varepsilon=1.753$ and the coefficient of skewness $C s=-0.393-$ by an order, see ([9], p.130).

\section{Results}

There exists a diagram of $\mathrm{K}$. Pearson distribution types, which is built in the coordinates $\beta_{1}=$ $(C S)^{2}$ and $\beta_{2}=\varepsilon+3$ [11]. Calculations have shown (Fig. 2) that the points placed on this diagram using coordinates $\bar{\beta}_{1}$ and $\bar{\beta}_{2}$ (averaged values within every of temperature zone over every series) appeared in the region corresponding to type I (beta distribution) near the line $2 \beta_{2}-3 \beta_{1}-6=0$, corresponding to type III, which is used to approximate empirical distributions in engineering hydrology. For the critical temperature zone $\left(1.6-7{ }^{\circ} \mathrm{C}\right)$, the point is located in the region corresponding to type VI (beta-distribution of type II). Location of the points makes possible recognizing their coordinates $\beta_{1}$ and и $\beta_{2}$ (statistically significant) and identifying (irrespective of fractal diagnostics) the temperature range $1.6-7{ }^{\circ} \mathrm{C}$. This identification allows at least two conclusions.

The first is that the regulatory documents direct the designers to the Pearson distribution III, and establish involuntarily the correlation between the skewness and kurtosis coefficients by the formula $\varepsilon=(2 / 3) C s^{2}-2$ (it follows from the above expression for $\beta_{1}$ and $\beta_{2}$ and the equation of the line describing distributions of type III). However, as follows from Fig. 2, according to the field data, this correlation is by no means rigid even for the averaged observation series for $\beta_{1}$ and $\beta_{2}$ values. 


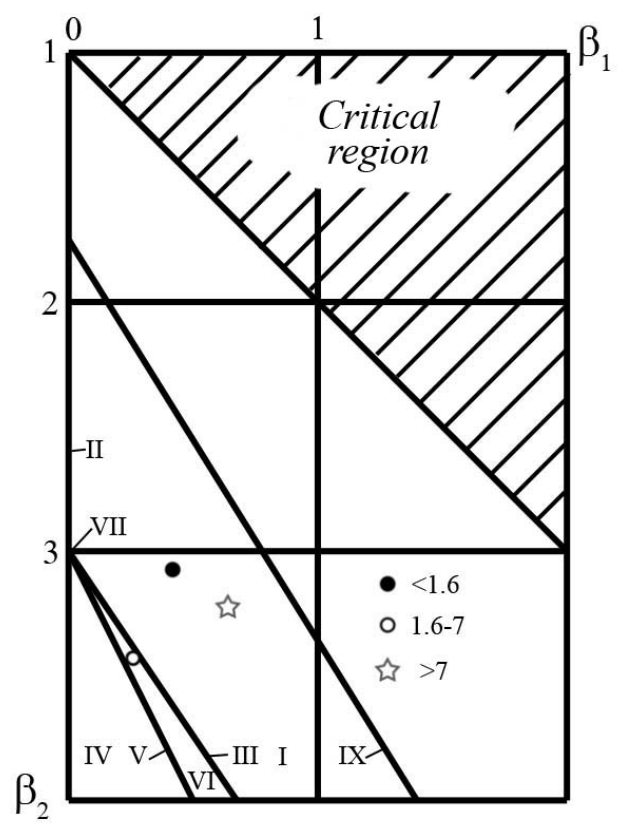

Figure 2. Part of a diagram of various distributions of the K. Pearson family (within the temperature range $-5-27^{\circ} \mathrm{C}$ ).

The second is as follows. If the graph in Fig. 1, $a$, indicates that the climatic component - air temperature - most complicates the process of runoff formation within in the range of $1.6-7^{\circ} \mathrm{C}$, the diagram in Fig. 2 shows that this climatic component clearly highlights the location of the hydrological representation. This is a set of parameters, $\beta_{1}$ and $\beta_{2}$, completely determined by the coefficients of skewness and kurtosis. Long-term river runoff, as well as climate, represents a natural resource for production functions of almost all water-dependent economy sectors. For example, in the construction design and operation of hydraulic structures, the long-term normal runoff is less important than the probability values of the design discharge that depends on the rate of tail lifting in probability distributions, i.e., on the higher moments that determine the coefficients of skewness and kurtosis. Therefore, the second conclusion is that ignoring kurtosis (or, rather its rigid binding to the coefficient of skewness, and the latter - to the coefficient of variation $C v$ : a fixed ratio $C s / C v$ is commonly used) actually means that the climatic component of natural resources is not accounted for.

Needless to say that such eliminating is done for objective reasons - insufficient time length of the runoff observation series, which brings significant errors in the estimates of $C s$ and $\varepsilon$. However, it can be demonstrated [10] that the numerical evaluations of $C s$ and $\varepsilon$ become steady if the length is 40-50 years (for $C s$ ) and 60-70 years (for $\varepsilon$ ). Fig. 3 demonstrates typical dependences of the examined coefficients on the number of series members. If we evaluate the computational errors for $C s$ and $\varepsilon$ only by the stabilized sections of such dependencies (approximately the last 30 years of observations), their computational error becomes comparable with that of the norm. 


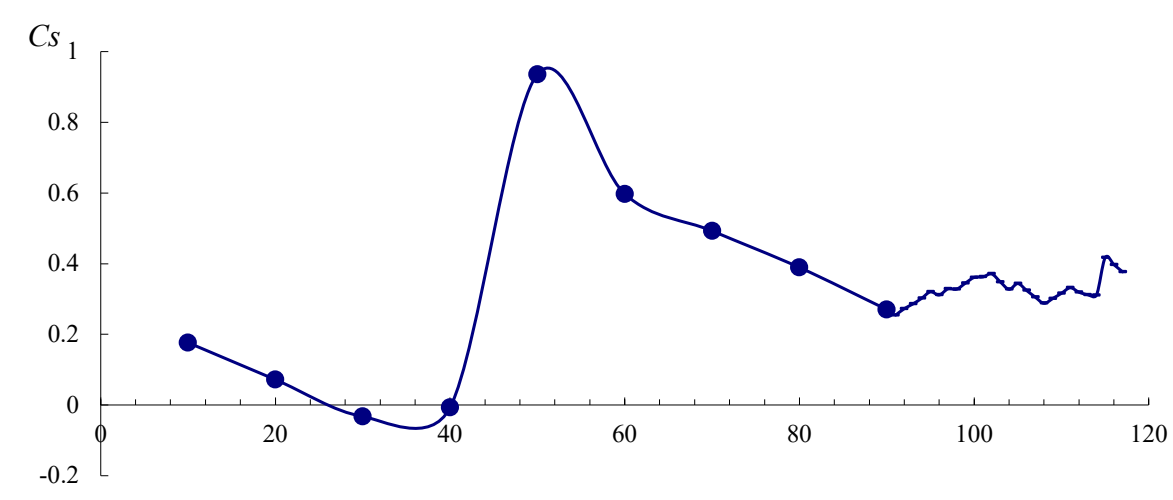

a

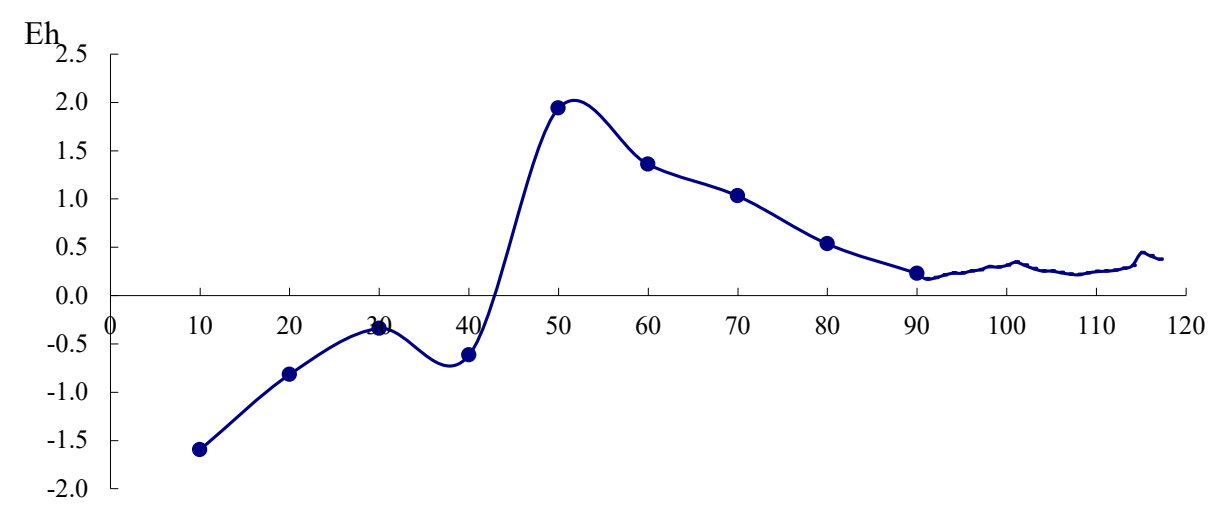

b

Figure 3. Dependence of the coefficients of skewness (a) and kurtosis (b) on the number of series members used in the calculations (r. Loire - st. Blois, France).

Thus, the geographic pattern identified in this article, which links climatic and hydrological components of the natural resources is not of cognitive interest exclusively, but can also be implemented in practice (the latter does not mean a call for the immediate revision of regulatory documents).

\section{Conclusions}

Relying on the earlier identified regular pattern of the influence of air temperature on the fractal dimension of the river runoff series, this article demonstrates, what exactly can serve as a visual image of the phenomenon that generates such linking (unimodal peaked probability density distributions with positive skewness values). It is empirically established that statistical distributions of long-term average annual runoff series for the temperature zone with prevailing high values of the fractal dimension correspond to beta-distributions of type II and when plotted on the K. Pearson diagram this type is distinguished from probability density curves in other temperature zones by skewness (statistically significant). As demonstrated, the discussed geographic pattern presents both cognitive and practical interest.

\section{Conflict of Interest}

The authors declare that there is no conflict of interest. 


\section{Acknowledgements}

This study was supported by the grant issued by the Ministry of Education and Science of the Russian Federation under the state contract No. 5.6293.2017/8.9 "Sensitivity of the long-term river runoff and major water-dependent sectors of the economy to climate change".

\section{References}

[1] Set of Rules 33-101-2003, Determination of the main calculated hydrological characteristics, Moscow, State Building of Russia, 2004. (In Russian).

[2] V.V. Kovalenko, Partially infinite modelling: basis, examples, paradoxes, Polytechnic, St. Petersburg, Russian Federation, 2005. (In Russian).

[3] V.I. Naidenov, Nonlinear dynamics of surface land waters, Science, Moscow, Russian Federation, 2004. (In Russian).

[4] V.V. Kovalenko et al., Forecasting the changes in the fractal dimension of the long-term river flow, Geography and Natural Resources. 4 (2008) 136-143. (In Russian).

[5] F. Takens, Detecting strange attractors in turbulence, Lect. Notes in Match, Berlin, Springer, 1981, pp. 336-381.

[6] V.V. Kovalenko, E.V. Gaidukova, Influence of climatological norms of the surface air temperature on the fractal dimensionality of the series of long-term river discharge, Doklady Earth Sciences. 439(2) (2011) 1183-1185. Doi: 10.1134/S1028334X1108023X.

[7] S.N. Kritsky, M.F. Menkel, On the techniques for studying random fluctuations of river runoff, Proceedings of NRU GUGMS. 4 (1946) 3-32. (In Russian).

[8] V.V. Kovalenko, Theoretical and experimental substantiation of the correlation between the fractal dimension of long-term flow series and the climatic norm of surface air temperature, Doklady Earth Sciences. 444(2) (2012) 666-670. Doi: 10.1134/S1028334X12060232.

[9] A.K. Mitropolsky, Technique of statistical calculations, Science, Moscow, USSR, 1971. (In Russian).

[10] V. Kovalenko, E. Gaidukova, A. Kachalova, An opportunity of application of excess factor in hydrology, Hydrology and Earth System Sciences Discussions. 9 (2012), 13635-13649. Doi: 10.5194/hessd-9-13635-2012.

[11] V.I. Tikhonov, Statistical radio engineering, Radio and Communication, Moscow, USSR, 1982. (In Russian). 